\title{
LOS USOS ATENUANTES DEL APROXIMATIVO CASI: RELACIÓN ENTRE CONTENIDO PROPOSICIONAL Y UNIDADES DEL DISCURSO*
}

\author{
MITIGATING-CASI: RELATION BETWEEN PROPOSITIONAL \\ CONTENT AND DISCOURSE UNITS
}

\author{
AdriÀ PARDo Llibrer \\ Universitat de València \\ Grupo Val.Es.Co. \\ Adria.Pardo@uv.es
}

Recibido: 20/12/2016

Aceptado: 02/05/2017

\begin{abstract}
Resumen
El aproximativo casi entraña la inversión polar de su proposición, sin embargo, los usos considerados expletivos presentan valores atenuantes. Este trabajo complementa con un estudio de corpus la descripción teórica del uso expletivo-atenuante en relación con la unidad discursiva de casi en el habla coloquial. La unidad que ocupa en el discurso determina su uso atenuante y supone una prueba efectiva de su naturaleza semántica, al tiempo que aclara ciertas cuestiones de la bibliografía deductiva.

PALABRAS CLAVE: casi, atenuación, contenido proposicional, unidades discursivas, aproximativos, inercia asertórica.
\end{abstract}

\begin{abstract}
This paper explores the mitigating function of Spanish casi and its relation to the discourse units casi fulfills in spoken language. Casi can either entail a polar inversion of the proposition it has scope over or constitute a case of mitigation (expletive use). This paper implements the theoretical description with corpus-based examples. All examples are divided into discourse units, providing an account of mitigating values and clarifying some controversial aspects found in the literature on approximatives.

KEY WORDS: casi, mitigation, propositional content, discourse units, approximative adverbs, assertoric inertia.
\end{abstract}

\footnotetext{
* Este artículo se enmarca dentro del proyecto FF120216-77841-P, UDEMADIS (Unidades discursivas para una descripción sistemática de los marcadores del discurso en español), financiado por el Ministerio de Economía y Competitividad.
}

Para citar este artículo / To cite this article: Pardo Llibrer, Adrià (2018): Los usos atenuantes del aproximativo casi: relación entre contenido proposicional y unidades del discurso. García Ramón, Amparo y Soler Bonafont, María Amparo (Eds.): ELUA: Estudios de atenuación en el discurso, Anexo IV, págs. 33-56.

Enlace / Link: http://dx.doi.org/10.14198/ELUA2018.Anexo4.03 


\section{INTRODUCCIÓN}

El presente trabajo tiene por objetivo explicar los usos atenuantes del adverbio aproximativo casi en relación con la unidad discursiva que este ocupa. La forma casi constituye, desde hace treinta años, el principal objeto de estudio de toda una línea de investigación desarrollada en pragmática teórica (Li 1976; Sadock 1981; Ziegeler 2000, 2010; Amaral 2006; Horn 2009, 2011; Amaral y Del Prete 2010). Estos análisis son mayoritariamente deductivos: no acuden a ejemplos extraídos de corpus con los que dar cuenta de los casos que escapan a la introspección. Por ello, este estudio recurre a ejemplos reales de conversaciones coloquiales para analizar aquellos usos de casi denominados expletivos. Consideramos estos usos expletivos como propiamente atenuantes y pensamos que la naturaleza semántica de este aproximativo, así como los problemas teóricos que plantea, pueden dilucidarse mediante una segmentación en unidades discursivas (para lo que nos servimos del modelo de Grupo Val.Es.Co.). Este artículo introduce, siguiendo a Pons y Schwenter (2011), significado (§ 2.1.1.) y tipos de casi (§ 2.1.2). Seguidamente, exponemos el problema del denominado componente polar en los usos atenuantes (§ 2.2.1.) y explicamos su desactivación desde la tesis semántico-pragmática de la inercia asertórica (§ 2.2.2). Por último (§ 2.3.), introducimos el modelo Val.Es.Co. de unidades discursivas, a fin de relacionar la noción de contenido proposicional con la naturaleza semántica de casi. Una vez presentados estos contenidos, enunciamos la hipótesis de nuestro estudio $(§ 3)$ : debido a su naturaleza semántica asertóricamente inerte, los usos atenuantes de casi (pragmáticos) tienen lugar en unidades discursivas carentes de contenido proposicional. A ello sigue un estudio de corpus (§ 4.1.) y el análisis de algunas ocurrencias (§ 4.2.) con las que ejemplificamos la relación entre usos atenuantes, unidades y contenido proposicional. Las conclusiones cierran el artículo $(\S 5)$.

\section{ESTADO DE LA CUESTIÓN}

En este apartado, presentamos los componentes fundamentales del significado de los aproximativos (§ 2.1.1.), así como los diferentes tipos de casi (§ 2.1.2.). Asimismo, exponemos cómo se ha estudiado esta forma: los dos enfoques que centran el debate sobre su descripción (§ 2.2.1.) y la explicación de la inercia asertórica que aquí defendemos ( $\S$ 2.2.2.). Finalmente, introducimos el modelo Val.Es.Co. (§ 2.3.).

\subsection{Significado y tipos de casi}

\subsubsection{Componentes próximo y polar}

La bibliografía pragmática (Sadock 1981; Ziegeler 2000; Horn 2009; Pons y Schwenter 2011), así como algunos trabajos de semántica formal (Aranovich 1995, Sevi 1998), distinguen dos componentes fundamentales de significado para la descripción del aproximativo casi (Cuadro 1):

\begin{tabular}{|l|c|c|}
\hline & COMPONENTE PRÓXIMO & COMPONENTE POLAR \\
\hline $\begin{array}{l}\text { Casi me muero } \\
\text { (casi } \text {-P })\end{array}$ & $\begin{array}{c}\text { he estado cerca de morir } \\
(\mathrm{P})\end{array}$ & $\begin{array}{c}\text { (pero) NO muero } \\
(\neg \mathrm{P} ’)\end{array}$ \\
\hline
\end{tabular}

Cuadro 1. Componentes próximo y polar. 
El componente próximo denota un proceso de culminación a la realización del predicado sobre el que casi tiene ámbito ('cercanía a morir'), mientras que el componente polar supone una inversión de los valores de verdad de la proposición en que el aproximativo se encuentra ('NO muero').

Asimismo, el componente próximo puede dividirse en dos subcomponentes (Ducrot 1982) (Cuadro 2):

\begin{tabular}{|l|l|c|}
\hline & COMPONENTE PRÓXIMO & COMPONENTE POLAR \\
\hline El euro vale casi un dólar, es caro & $\begin{array}{l}\text { El euro está cerca de valer un dólar } \\
\text { ASCENDENTE: } \uparrow P \text { (se aproxima al dólar) }\end{array}$ & $\begin{array}{c}\text { no vale un dólar } \\
(\neg \mathrm{P} \text { ') }\end{array}$ \\
\hline El euro vale casi un dólar, es barato & $\begin{array}{l}\text { El euro está cerca de NO valer un dólar } \\
\text { DESCENDENTE: } \downarrow P \text { (se distancia del dólar) }\end{array}$ & \\
\hline
\end{tabular}

Cuadro 2. Componente próximo ascendente y descendente.

Cuando casi denota un proceso de culminación hacia la realización del predicado sobre el que tiene ámbito, presenta un componente próximo ascendente ( $\uparrow$ P). Pero, cuando casi denota un proceso de culminación hacia la no-realización del predicado $(\uparrow \neg \mathrm{P})$, el componente próximo es descendente $(\downarrow P)$; ya que, si bien denota proximidad, en lugar de cercanía connota distanciamiento.

\subsubsection{Tipos de casi}

Según presente una u otra combinación de sus componentes próximo (CPROx) y polar (CPOL), se pueden organizar las diferentes realizaciones de casi en cuatro tipos (siguiendo a Pons y Schwenter 2011):

a. Uso CANÓNICO. Es el tipo más frecuente en el habla:

(1) El libro cuesta casi 15 euros.

a. CProx: cercano a costar 15 euros

b. CPOL: (pero) NO cuesta 15 euros

Presenta un componente próximo ascendente (1a) e invierte el valor de verdad de su proposición (1b):

(1') El libro cuesta casi 15 euros.

a. CProx: ascendente ( $\uparrow \mathrm{P})$

b. CPOL: activado ( $\neg$ P')

b. Uso PSEUdOCANÓNICO. Constituye la contrapartida descendente del casi canónico:

(2) El libro cuesta casi 15 euros, es barato.

a. Cprox: cercano a no costar 15 (= se distancia de costar 15)

b. CPOL: (pero) NO cuesta 15 euros 
La circunstancia de 'ser barato' (2) frente a, p. ej., 'ser caro' ( $c f . \S 1.1)$ supone un co(n) texto que restringe el componente próximo a una lectura descendente. Se distancia la realización del predicado (2a), pero se conserva el componente polar activado (2b):

(2') El libro cuesta casi 15 euros, es barato.

a. CPRox: descendente $(\downarrow P)$

b. CPoL: activado ( $\left.\neg \mathrm{P}^{\prime}\right)$

c. Uso ATENUANTE. Los usos anteriores constituyen la imagen especular de un mismo fenómeno: la inversión polar de la proposición en que casi se encuentra. Sin embargo, hay casos en que el componente polar aparece desactivado:

(3) Casi paga tú y luego hacemos cuentas.

a. CPRox: distanciamiento del acto de 'pagar'

b. CPOL: sí pagas tú

Este uso se denomina generalmente casi expletivo (Ziegeler 2010, 2016), pues no invierte la polaridad y, a nivel proposicional, no es imprescindible para entender su significado formal ${ }^{1}$. Sin embargo, a nivel discursivo, considerar este uso como expletivo presupone un sentido idéntico para los siguientes enunciados:

(3) Casi paga tú y luego hacemos cuentas.

(3') Paga tú y luego hacemos cuentas.

Al no desatar polaridad, el significado de (3) es proposicionalmente idéntico a (3'). Así, el componente polar en ( $3 \mathrm{~b})$ está desactivado y su componente próximo es descendente ( $3 \mathrm{a})$ :

(3") Casi paga tú y luego hacemos cuentas.

a. Cprox: descendente $(\downarrow P)$

b. CPOL: desactivado (P')

Al no producirse una inversión polar de $P(3 \mathrm{~b})$, la relevancia lingüística del uso de casi en un ejemplo como (3) es más pragmática que semántica, pues el componente próximo descendente de casi conlleva un distanciamiento de $P$ sin negar la proposición (casi-P $\rightarrow \mathrm{P}^{\prime}$ ). La diferencia discursiva entre un sentido más modal (3) y otro más neutro (3') muestra cómo el casi denominado expletivo tiene alcance sobre el enunciado y no sobre la proposición. Como recurso pragmático, supone una atenuación con respecto al hecho de 'pagar' que no se produce en (3'), aunque el predicado esté afirmado en ambos casos. Así, casi funciona aquí como elemento de una estrategia atenuante (Albelda 2010): el hablante de (3) protege su imagen ante un acto de habla potencialmente descortés, como es pedirle a su interlocutor que pague por él.

1 Cabe señalar que, en su estudio, Pons y Schwenter (2011: 170-178) hacen referencia a los valores “epistémicos e ilocutivos" de casi, señalando igualmente su aparición en enunciados propios de la cortesía comunicativa. Por este motivo, mantenemos el término atenuante como etiqueta general a falta, por el momento, de un estudio de corpus más detallado. 
Ante la falta de concreción del término expletivo, consideramos más adecuada la etiqueta atenuante, pues remite a una función específica del uso de casi con el componente polar desactivado, cuyo uso (frente a la mera omisión) es recurrente dentro de actos de habla marcados cortésmente.

d. Uso invertido. Es el menos común, presente (al menos) en el español de la Comunidad Valenciana (Schwenter 2002). A modo de ejemplo: supongamos que una madre enfadada con su hijo quiere dirigirse enérgicamente a este y, a la hora de llamarlo, enumera los nombres de todos sus hermanos antes de dar con el vocativo correcto. Bajo estas circunstancias, el hijo aludido puede contestar:

(4) ¡Casi lo dices!

a. Cprox: has estado cerca de decirlo

b. Crol: (y, de hecho) sí lo has dicho

Es un uso invertido porque coincide con el uso canónico en su proximidad ascendente (4a), aunque difiere en la inversión de la polaridad (4b):

(4') ¡Casi lo dices!

a. CPROX: ascendente $(\uparrow \mathrm{P})$

b. CPOL: desactivado ( $\left.\mathrm{P}^{\prime}\right)$

El casi invertido constituye un uso sujeto a fuertes restricciones pragmáticas: expresa un sentido irónico codificado entre hablantes de una misma variedad de habla y en situaciones de marcado carácter interpersonal.

En conclusión, de acuerdo con la categorización de Pons y Schwenter (2011) encontramos una cuádruple clasificación (Cuadro 3):

\begin{tabular}{|l|c|c|}
\hline \multicolumn{1}{|c|}{ USOS } & COMPONENTE POLAR & COMPONENTE POLAR \\
\hline Canónico & ascendente $(\uparrow \mathrm{P})$ & $\left(\neg \mathrm{P}^{\prime}\right)$ \\
\hline Pseudocanónico & descendente $(\downarrow \mathrm{P})$ & $\left(\neg \mathrm{P}^{\prime}\right)$ \\
\hline Atemuante & descendente $(\downarrow \mathrm{P})$ & $\emptyset$ \\
\hline Invertido & ascendente $(\uparrow \mathrm{P})$ & $\varnothing$ \\
\hline
\end{tabular}

Cuadro 3. Combinatoria de componentes.

En adelante, este trabajo se ocupa del análisis del casi redefinido como atenuante en contraposición con los usos (pseudo)canónicos ${ }^{2}$, dejando a un lado cuestiones relativas al uso invertido ${ }^{3}$.

2 En adelante, bajo el término (pseudo)canónico agrupamos los usos canónicos y pseudocanónicos. Ambos usos, independientemente de su componente próximo, se reconocen por la activación de su componente polar, en contraposición a los usos atenuantes.

3 El denominado uso invertido requiere futuros estudios sobre realizaciones semejantes en otras variedades aparte del español de Valencia. Solo la sucinta descripción ofrecida en $\S 2.1 .2$. $d$ ya evidencia las diferentes posibilidades que, en función de sus componentes, puede realizar casi. 


\subsection{Casi en la investigación en aproximativos}

Todas las realizaciones del aproximativo casi presentan uno u otro tipo de proximidad, pero solo los usos (pseudo)canónicos desencadenan polaridad. Los usos atenuantes, por el contrario, tienen un valor más pragmático y no invierten el valor de verdad de su proposición. El componente polar es, pues, el fenómeno que más atención merece a los pragmatistas; no solo porque de una proposición formalmente positiva (casi-P) se desprende una lectura negativa de la misma (casi-P $\left.\rightarrow \neg \mathrm{P}^{\prime}\right)$, sino también porque este proceso inferencial se suspende en aquellos usos pragmáticamente atenuadores.

\subsection{1. ¿Qué es el componente polar?}

La naturaleza del componente polar ha centrado el debate lingüístico sobre los aproximativos. Este se ha explicado tanto desde enfoques discursivos (Sadock 1981, Ziegeler 2000, 2010) como en términos semánticos (Sevi 1998, Horn 2009, 2011). Este artículo no se ocupa en profundidad de las diferentes líneas que han tratado la polaridad en los aproximativos, sin embargo, conviene esbozar los dos enfoques principales que analizan el componente polar. Por una parte, un enfoque estrictamente discursivo, en línea con la Radical Pragmatics (Sadock 1981, Ziegeler 2000); por otra parte, un enfoque más semántico-pragmático (desarrollado en los trabajos de Horn (2002, 2009, 2011)).

El enfoque discursivo de la Radical Pragmatics entiende el componente polar como la instancia lingüística que se sigue inmediatamente del componente próximo. Un ejemplo como:

(5) Casi me manché.

a. CProx: cerca-de-mancharme $(\uparrow \mathrm{P})$

b. CPOL: (pero) No me manché ( $\neg$ P')

revela, en primer término, un significado léxico del aproximativo casi. Este significado léxico es lo que se denomina componente próximo; el cual, al denotar un proceso de culminación hacia el predicado que modifica (5a), está significando proximidad al acto de 'mancharse'. Sin embargo, este proceso de proximidad progresiva supone una culminación irrealizada. Si, a nivel gramatical, casi modifica aspectualmente su predicado (De Miguel 1999, Fernández Leborans 1999, Kovacci 1999), el proceso (de carácter imperfectivo) de proximidad al acto de 'mancharse' es incompatible con la realización (perfectiva) del mismo. De esta irrealización del predicado se infiere, a nivel pragmático, la no-culminación del acto de 'mancharse'; lo que, de acuerdo con la lógica conversacional (Grice 1975, Levinson 2000) constituye una implicatura conversacional generalizada (en adelante ICG) de base Q:

(5') Casi me manché.

a. CPRox: cerca-de-mancharme ( $\mathrm{Q}^{+>}$no completo la acción de 'mancharme')

b. CPol: (no-culminación $++>$ ) No me manché

Como señala Sadock (1981: 270), aunque “casi [almost, en el original] resulta complicado de analizar desde el punto de vista de aquello que expresa, es simple en términos de contenido 
convencional". En otras palabras: el carácter léxico-aspectual de casi y su funcionamiento a nivel pragmático como componente próximo es especialmente complejo de reconstruir en tanto que implicatura (categoría difusa), pero es fácilmente identificable en la lengua al uso como un elemento de valor negativo, dado su componente polar (categoría discreta).

El uso de casi implica un contenido (P) poco informativo (5'a), una violación de la máxima de cantidad griceana de la que se deduce, como correlato comunicativo $\left(5^{\prime} \mathrm{b}\right)$, la negación del predicado modificado $\left(\neg \mathrm{P}^{\prime}\right)$. De esta manera, el componente polar se entiende como una forma alternativa de negar $P$, resultado de un proceso pragmático de recuperación inferencial (discourse-worked, Ziegeler 2000: 1744). Así, el componente próximo constituiría el significado base de casi, mientras que el componente polar sería un fenómeno $a$ posteriori puramente discursivo.

A partir de Sadock (1981), el componente polar se considera resultado de una ICG. Esta postura se perfila posteriormente con los trabajos de Ziegeler (2000, 2010), quien considera que el uso de casi tiene lugar en enunciados contrafactuales. Un ejemplo paradigmático de enunciado contrafactual son las oraciones condicionales potenciales (Cuadro 4):

\begin{tabular}{|l|l|l|l|}
\hline Si estudiaras más, & CONOCIMIENTO FACTUAL & HiPÓTESIS & CONTRAFACTUALIDAD \\
\cline { 2 - 4 } sacarías mejores notas. & no estudias lo suficiente & buenas notas & sacas malas notas \\
\hline
\end{tabular}

Cuadro 4. Condicionales como enunciados contrafactuales.

Estos casos ejemplifican las restricciones propias de los enunciados contrafactuales (Ziegeler 2000):

a. Por un lado, dada la dependencia del contexto, un conocimiento real de los hechos (el hablante es consciente de que su interlocutor no estudia lo suficiente).

b. Por otro lado, instructores morfosintáctictos que ayudan al oyente en su evaluación de estos hechos (en el caso de las condicionales, el uso de los modos subjuntivo y condicional).

En este sentido, las ICG que tienen lugar en enunciados contrafactuales funcionan propiamente como implicaturas contrafactuales (en adelante, ICF). Así, de manera similar a como ocurre en las condicionales, las restricciones de la contrafactualidad se adecúan también a los enunciados con casi (Cuadro 5):

\begin{tabular}{|l|l|l|l|}
\hline $\begin{array}{l}\text { Casi marca el Barça y } \\
\text { pierdo la apuesta. }\end{array}$ & CONOCIMIENTO FACTUAL & HIPÓTESIS & CONTRAFACTUALIDAD \\
\cline { 2 - 4 } & CASI marca el Barça & perder la apuesta & $\begin{array}{l}\text { gano la apuesta } \\
\text { (casi-marcar } \rightarrow \text { NO-marcar })\end{array}$ \\
\hline
\end{tabular}

Cuadro 5. Casi y enunciados contrafactuales.

Teniendo esto en cuenta, el componente polar, en tanto que ICF, cumpliría los requisitos propios de un enunciado contrafactual. Así pues, en un enunciado como "Casi marca el Barça”, el uso de casi denota un predicado del tipo 'próximo a marcar' ( $\uparrow \mathrm{P})$ del cual, en principio, se sigue la hipótesis (positiva) 'ha marcado' (P’). Sin embargo, el hecho de que 
casi tenga lugar en un enunciado contrafactual implica que, del predicado que modifica, se desprenda la hipótesis contraria al conocimiento factual del mundo: 'el Barça no ha marcado’ ( $\neg$ P’). Esta inferencia pragmática es lo que se denomina componente polar: la ICF permite recuperar la hipótesis opuesta a $P$ ('marcar') que, en su correlato comunicativo $(++>\neg \mathrm{P}$ '), se lee como una negación ('no ha marcado'), y cuyo instructor morfosintáctico es el adverbio casi.

Extender la explicación de ICG a la de ICF puede parecer una matización formal innecesaria para los fines de este apartado introductorio. No obstante, la consideración del componente polar como ICF propone una explicación pragmática para los usos atenuantes de casi (expletivos en Ziegeler (2016)). El hecho de que haya casos en que el aproximativo casi no presente una inversión polar se debería a que el componente próximo no desata una ICF de la que inferir un componente polar, puesto que no tendría lugar en contextos contrafactuales. La ICF se cancelaría al darse en un contexto despreferido, ya que "el hablante es consciente del impacto emocional en el receptor al enunciar cercanía a lo desafortunado" (closeness to misfortune en el original, Ziegeler (2016: 21)). El uso de casi en un contexto despreferido supone un conocimiento fáctico (real) del mundo por parte del hablante, pero casi no actúa como instructor para la hipótesis contraria $\left(\neg \mathrm{P}^{\prime}\right)$ porque el contenido $(\mathrm{P})$, aunque polémico, no puede ser negado (ya que el conjunto del enunciado perdería coherencia). Así, si el contexto es despreferido, se suspende tanto el enunciado contrafactual como el consiguiente componente polar. Por lo que este uso de casi se concibe como expletivo (en nuestra opinión, atenuante, dado su valor modalizador, vid. § 2.1.2 a).

En resumen, en la investigación sobre aproximativos, la línea teórica estrictamente pragmática, iniciada por Sadock (1981) en la Radical Pragmatics y continuada por Ziegeler (2000), contempla el componente polar como un fenómeno propio del discurso. Está relacionado con la semántica de casi pero no constituye una parte fundamental de esta. La inversión polar estaría sujeta al contexto, tal y como vendría a probar la existencia de usos atenuantes.

\subsubsection{La inercia asertórica: justificación teórica y problemas}

Como alternativa al enfoque estrictamente pragmático de la ICF, ciertos trabajos (Horn 2002, 2009, 2011) definen el componente polar como una relación lógica de entrañamiento. El uso de casi en una proposición dada entraña un componente polar, como parte sustancial de su significado. Esto es: el uso de casi es indisociable de su componente polar y, en la medida en que está entrañado, es una parte tan fundamental de su significado como el componente próximo:

(6) Casi me manché.

a. CProx: significa 'cercanía-a-mancharme' $(\uparrow \mathrm{P})$

b. CPoL: entraña 'NO me manché' $(\Rightarrow \neg P$ ')

Como resultado de un entrañamiento, el componente polar se sitúa también en un nivel semántico. Mientras que el componente próximo (6a) denota un significado léxico determinado (ascendente o descendente), el componente polar (6b) repercute sobre el significado formal (las condiciones lógico-veritativas de la proposición); siendo ambas las dos caras de 
una misma significación, y no el segundo resultado del primero. A diferencia de la explicación de la ICF, que considera el componente polar como una realización pragmática del componente próximo (Cuadro 6):

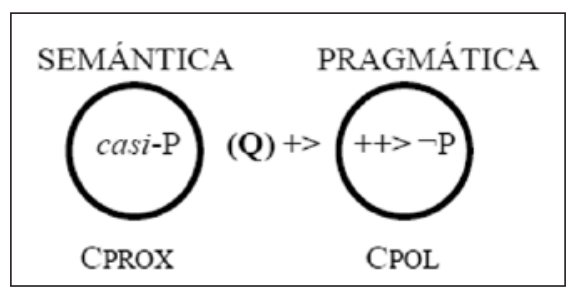

Cuadro 6. Componente polar según Radical Pragmatics.

La tesis de la inercia asetórica toma ambos componentes como parte del significado de casi; considerando el componente polar como material semánticamente entrañado, aunque de manera inerte (Cuadro 7):

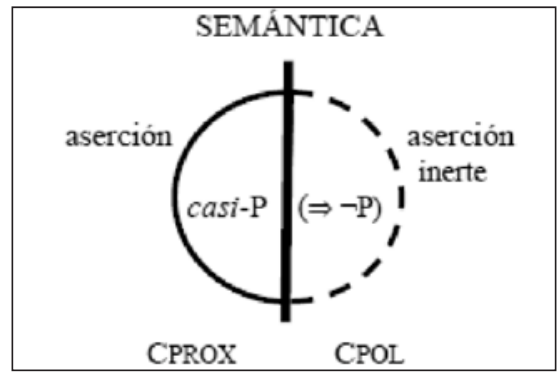

Cuadro 7. Componente polar y aserción inerte.

Como aserción inerte, el componente polar se considera un entrañamiento sin sustancia semántica (Horn 2002). Es decir, es un componente que funciona por inercia (pues siempre está entrañado), pero depende de otros elementos lingüísticos semánticamente sustanciales para activarse. De este modo, el entrañamiento polar contenido en su significado (el componente polar) se realiza igualmente en el discurso (inversión polar) (Cuadro 8):

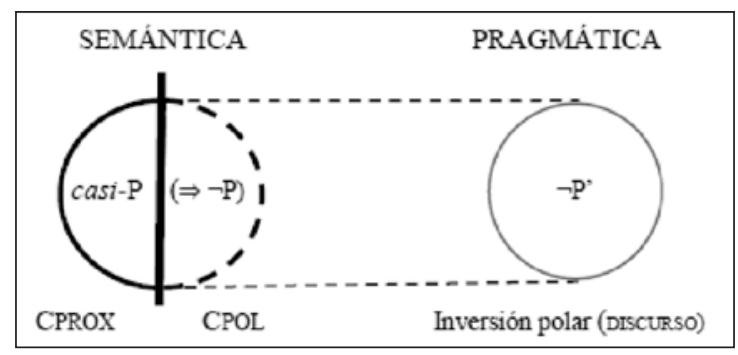

Cuadro 8. Componente polar (semántica) / inversión polar (realización discursiva). 
Situar ambos componentes en el nivel semántico implica considerar tanto proximidad como polaridad como los dos elementos definitorios de la naturaleza semántica de casi. Ahora bien, el componente próximo tiene lugar en todas las realizaciones de casi (vid. $\S$ 2.1.2.), mientras que el componente polar, pese a estar entrañado, no siempre invierte la polaridad de la proposición. Luego, los usos (pseudo)canónicos de casi invierten los valores de verdad, pero los usos atenuantes no (Cuadro 9):

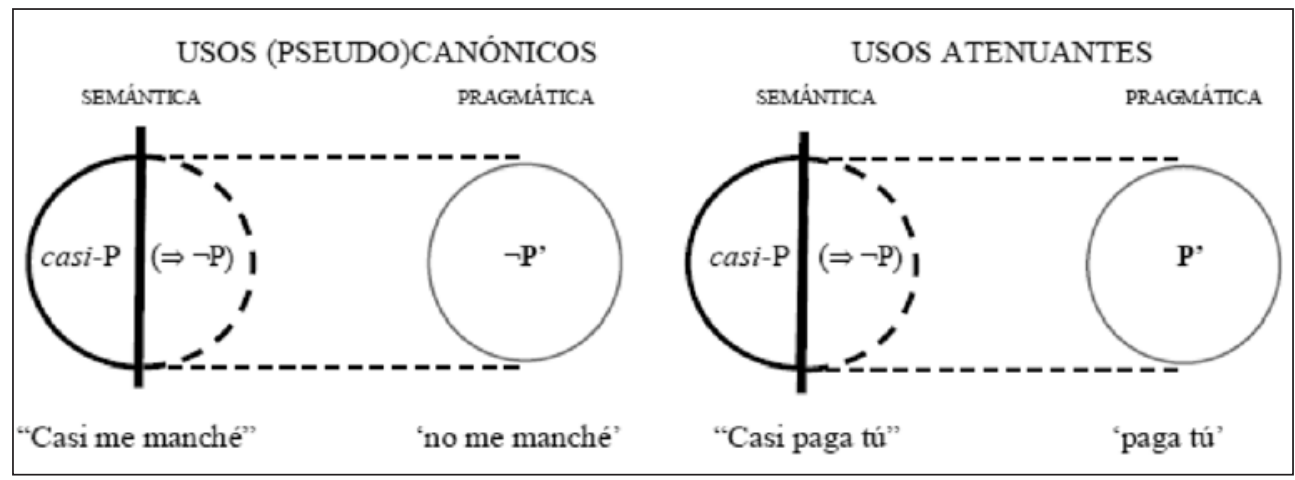

Cuadro 9. Usos canónicos y pseudocanónicos frente a usos atenuantes.

De acuerdo con Horn (2011), esto se debe a que el componente polar está entrañado, pero constituye un entrañamiento asertóricamente inerte. Según este análisis, el componente polar es una parte necesaria (entrañada) del significado de casi pero, al carecer de sustancia semántica, se aserta de manera inerte. Es por esto que, en ocasiones, como ocurre en los usos atenuantes, no basta con el mero empleo de casi para que siempre se produzca una inversión polar. La inercia asertórica sugiere que el componente polar está presente como parte del significado de los aproximativos, pero depende de la sustancia semántica de otros elementos para activarse o desactivarse (esto es, para invertir el valor de verdad de la proposición).

\subsubsection{Justificación teórica}

De entrada, la explicación del entrañamiento asertóricamente inerte parece poco económica. El componente polar es concebido como un mecanismo lingüístico indisociable de casi (pues está entrañado), el cual siempre (por inercia) subyace a su uso (asertado); tanto cuando invierte la polaridad (activado) como cuando no (desactivado). Por ello, exponemos brevemente las tres razones principales por las que se puede deducir que la inercia asertórica sería el lugar semántico que corresponde a los aproximativos:

a. En primer lugar (siguiendo a Horn (2002)), si se considera el componente polar como la implicatura inferida de un acto incompleto, la locución adverbial no del todo (not quite, en el original) debería funcionar como correlato sinonímico de casi. Ambos denotan no-culminación del predicado y ambos entrañan negación. Sin embargo, si el compo- 
nente polar resulta de una implicatura generalizada por la máxima de cantidad, deberían darse inferencias semejantes para ambos adverbiales. Por el contrario (Horn 2002: 63):

(7) Lamento que casi murieras ("it's too bad you almost died").

a. $\mathrm{Q}+>$ 'No moriste'.

b. $++>$ 'Ahora te toca hacer convalecencia'.

(8) Lamento que no murieras del todo ("it's too bad you didn't quite die").

a. Q+> 'No moriste'.

b. $++>$ 'Ahora tengo que rematarte yo'.

Las implicaturas varían en función de si utilizamos casi (7) o no del todo (8). El adverbial no del todo entraña $\neg P$, pues es en sí mismo un compuesto negativo; mientras que solo a casi se le presupone un componente polar. Si consideramos el componente polar un entrañamiento, la necesidad del estado semánticamente intermedio de la inercia asertórica se justifica porque solo podemos comparar ambas formas desde el nivel que comparten. Y puesto que a no del todo no se le presupone una polaridad susceptible del análisis pragmático, dicho nivel común es el semántico.

b. En segundo lugar, entender el componente polar como un entrañamiento asertóricamente inerte, en nuestra opinión, permite explicar de manera teórica los usos considerados expletivos en la bibliografía:

(9) Casi paga tú y hacemos cuentas.

a. CProx: distanciamiento de 'pagar'.

b. Cpol: (pero) paga tú.

Al entender que el componente polar está siempre entrañado, hay que tomar la lectura positiva de (9b) como una realización inerte del componente polar, asertado pero no activado. Este, al no conllevar una inversión polar de la proposición, repercute sobre el enunciado desarrollando valores pragmáticos (uso atenuante).

c. Por último, los entrañamientos asertóricamente inertes carecen (según Horn (2002, 2011)) de sustancia semántica (y precisan la de otros elementos lingüísticos).

\subsubsection{Problemas teóricos}

La tesis del entrañamiento asertóricamente inerte está formalmente justificada para igualar ambos componentes al nivel semántico, pero a costa de sostener un alto nivel de conceptualización. Esto conlleva, en relación con las tres razones expuestas anteriormente, tres problemas teóricos:

a'. En primer lugar, considerar el componente polar como un entrañamiento asertóricamente inerte es admisible para igualar casi y no del todo al nivel semántico; pero ello no impide que (aun sin componente polar) no del todo tenga sus propios desarrollos pragmáticos. 
b'. Igualmente, concebir el componente polar como un entrañamiento inerte plantea que los usos atenuantes son una realización discursiva (relacionada pero independiente) del material semántico asertado. El componente polar en casi, al desactivarse y no desatar polaridad proposicional, implica una prominencia semántica del componte próximo descendente (así como de los valores atenuantes derivados en el nivel pragmático). El problema es que, si los usos atenuantes son discursivos, también deben serlo los casos en que el componente polar está activado y, por tanto, entrañamiento e inversión polar coinciden (los cuales, además, son la mayoría).

c'. Por último, recurrir a la noción de sustancia semántica, sin profundizar en su definición, supone más bien un problema añadido que una herramienta explicativa.

Como exponemos a continuación, estos problemas teóricos pueden resolverse si atendemos a los usos atenuantes del aproximativo casi desde la segmentación discursiva.

En conclusión, según la línea teórica en que se desarrollan (bien pragmática radical o bien semántico-pragmática), hay dos tratamientos para los usos atenuantes de casi:

- Contrafactualidad. Según la icf, los usos atenuantes responden a cancelaciones de la hipótesis contraria cuando, en un contexto despreferido al hablante, el enunciado contrafactual queda suspendido.

- Inercia ASERTóRica. Según el entrañamiento asertóricamente inerte, los usos atenuantes están pragmáticamente marcados porque su componente polar está desactivado (y no conlleva inversión proposicional). La activación de este entrañamiento inerte no depende (como en Ziegeler 2016) de la predisposición cognitiva del hablante, sino de factores lingüísticos.

\subsection{El sistema Val.Es.Co. de unidades}

El modelo del grupo Val.Es.Co. ${ }^{4}$ propone un sistema de unidades discursivas para segmentar una conversación coloquial sin residuos. El objetivo de esta segmentación es ofrecer una sintaxis del habla (en términos de Narbona 1988) desde la que poder explicar ciertos fenómenos que oscilan entre los niveles gramatical y discursivo. Este modelo se ha aplicado en estudios sobre marcadores del discurso (Briz y Pons 2010; Estellés 2011), atenuación (Albelda 2005; Briz 2007) y gramaticalización (Estellés y Pons 2009; Salameh 2014). Pensamos, asimismo, que puede ser explicativo para ciertas cuestiones de la teoría de los aproximativos (especialmente en relación con la inercia asertórica). En este apartado, presentamos una síntesis introductoria al modelo.

\subsubsection{Jerarquía de unidades}

El sistema Val.Es.Co. se articula en torno a ocho unidades discursivas (discurso, diálogo, intercambio, alternancia de turnos, turno, intervención, acto, subacto). Estas unidades se

4 En este apartado, todas las referencias al modelo remiten a Briz y Grupo Val.Es.Co (2003) y Grupo Val.Es.Co. (2014). 
agrupan en dos niveles diferenciados (dialógico y monológico) que, a su vez, se organizan en tres órdenes distintos (dimensión estructural, social e informativa) (Cuadro 10):

\begin{tabular}{|l|l|l|l|}
\hline NIVEL & $\begin{array}{l}|c| \\
\text { ESTRUCTURAL } \\
\text { discurso } \\
\text { diálogo } \\
\text { intercambio }\end{array}$ & SOCIAL & INFORMATIVA \\
Dialógico & $\begin{array}{l}\text { intervención } \\
\text { acto }\end{array}$ & turno & subacto \\
\hline Monológico & & & \\
\hline
\end{tabular}

Cuadro 10. Niveles, órdenes, unidades.

El modelo establece una relación jerárquica y recursiva entre las diferentes unidades. Así, estructuralmente, la unidad dialógica superior es un discurso en su conjunto y, por debajo de este, se encuentra el diálogo, constituido a su vez por una sucesión de intercambios entre dos o más hablantes. En el orden social, este intercambio supone una alternancia de turnos, siendo el turno la unidad monológica del orden social. La diferencia entre un turno y una intervención es que, mientras que un turno debe ser aceptado por el otro interlocutor, la intervención queda delimitada estructuralmente por el cambio de emisor (llegue a constituirse como turno o no).

Los objetivos de nuestro estudio, pues, se centran en el nivel monológico. La intervención es aquella contribución del hablante a la conversación, cuyo constituyente inmediato es el acto. Toda intervención está constituida (al menos) por un acto, entendido este como la unidad mínima de acción e intención. Dos son los criterios propuestos para identificar actos:

a. Aislabilidad. Los actos son aislables: pueden funcionar como intervenciones independientes sin necesidad de estar acompañados de otros actos.

b. IdENTIFICABILIDAD. Los actos presentan límites formales (verbos ilocutivos, proformas, elementos extraproposicionales...) y prosódicos definidos.

Finalmente, un acto está constituido (al menos) por un subacto, que es la unidad informativa mínima, aunque podemos encontrar diferentes subactos en un mismo acto.

\subsubsection{Unidades y posiciones}

Las unidades acto y subacto del nivel monológico se diferencian jerárquicamente. El acto es el constituyente mínimo de una intervención. Así, un ejemplo como (10) sería una intervención con dos actos, porque ambos segmentos podrían funcionar como intervenciones (11):

(10) A: Bueno, si te parece pues me dejas hablar, ¿eh? Yo a ti siempre te escucho.

(11) a. A: \#Bueno, si te parece pues me dejas hablar, ¿eh?\#

b. A': \#Yo a ti siempre te escucho\#

Por debajo de esta división en actos encontramos dos tipos de subactos. Los subactos sustantivos (SS) y los subactos adyacentes (SA). Los ss se subdividen en subactos sustantivos directores (SSD), con el contenido proposicional nuclear; y en subactos sustantivos subor- 
dinados (sss), supeditados a la fuerza ilocutiva de los directores. Por otra parte, a los ss, se adscriben los SA, que pueden ser subactos adyacentes interpersonales (SAI), si implican interacción hablante-oyente; subactos adyacentes textuales (SAT), que organizan el discurso; y subactos adyacentes modalizadores (SAM):

(12) A: \#\{SAM Bueno, SAM $\}\{$ SSS si te parece SSS $\}\{$ SAT pues SAT $\}\{$ SSD me dejas hablar, $\mathrm{SSD}\}\{\mathrm{SAI} i \mathrm{eh}$ ?\# SAI $\}$

(13) A': \#\{SSD Yo a ti siempre te escucho SSD $\} \#$

Así, una intervención como (12) puede quedar segmentada siguiendo tanto criterios principalmente semánticos (ss) como criterios principalmente pragmáticos (SA).

De esta distinción en unidades menores al acto se sigue una relación de cuatro posiciones: inicial, media, final e independiente. Los SA mantienen una posición con respecto a los SS, por lo que, en (12), el SAM estaría en posición inicial de SSS; el SAT, en inicial de SSD; y el SAI, en final de SSD. Por su parte, la posición independiente solo la ocuparían los SSD; de ahí que la intervención (13) constituya un solo acto, mientras que en (12):

(12') \#\{SAM Bueno, SAM $\}\{$ SAT pues SAT $\}\{$ SSD me dejas hablar, SSD $\}\{$ SAI $¿ e h ? \#$ SAI $\}$ ??\#\{SAM Bueno, SAM $\}\{$ SSS si te parece SSS $\}\{$ SAT pues SAT $\}\{$ SAI $¿$ eh?\# SAI $\}$

solo el segmento $\{S S D$ me dejas hablar $S S D\}$ pueda ajustarse al criterio de aislabilidad.

\section{HIPÓTESIS}

La inercia asertórica funciona como un concepto definido en un marco teórico determinado: concluye en una explicación de los usos no-polares de casi como la realización de un entrañamiento carente de sustancia semántica. Este análisis puede complementarse con un estudio de carácter inductivo. Para ello, recurrimos al análisis de los usos atenuantes de casi dentro de la conversación coloquial. El modelo Val.Es.Co de unidades discursivas permite segmentar las posiciones que casi ocupa en el habla. Una explicación de los usos atenuantes basada en su estructura lingüística, en lugar de en consideraciones contextuales (Ziegeler 2016).

\subsection{Primera hipótesis: el casi atenuante ocupa una unidad sin contenido proposicional}

Los usos atenuantes se corresponden, en la investigación en aproximativos, con los usos expletivos. Si los usos (pseudo)canónicos presentan una inversión polar:

(14) Casi pago $60 €$ por esa entrada (casi-P $\Rightarrow \neg \mathrm{P} ')$

a. CProx: cercano a $60 €$

b. CPol: (pero) no 60

el uso denominado expletivo tendría un valor atenuante:

(15) Casi paga tú y luego hacemos cuentas (casi-P $\left.\Rightarrow \mathrm{P}^{\prime}\right)$

a. CPRox: distanciamiento de pagar

b. CPoL: pero pagas tú (desactivado) 
ya que presenta un componente próximo descendente (15a) que permite al hablante distanciarse de un acto que, si bien no está negado (15b), es potencialmente descortés. Así, encontramos dos tipos generales de casi en función de su polaridad:

(14') Casi pago $60 €$ por la entrada.

CPol-Activado ( $\rightarrow$ inversión del valor de verdad de la proposición).

(15') Casi paga tú y luego hacemos cuentas.

CPol-desactivado ( $\rightarrow$ valor pragmático, relacionado con su uso).

La cuestión de fondo al analizar los usos atenuantes es que, frente al funcionamiento de los usos (pseudo)canónicos (los más comunes), existen casos claramente no-polares:

(14") a. Casi pago $60 €$ [iqué caro!]

b. Casi pago $60 €[\mathrm{y}$, aunque sea más caro, ya me olvido]

Mientras que los usos (pseudo)canónicos (14"a) repercuten sobre la proposición, los usos atenuantes (14”b) lo hacen sobre el enunciado. Esta distinción entre el alcance semántico de (14"a) y pragmático de (14"b) no aclara, sin embargo, por qué unas veces el componente polar se activa y otras no. Si consideramos el componente polar como un entrañamiento asertóricamente inerte, los usos (pseudo)canónicos serían los que poseen sustancia semántica. Es decir, si los usos (pseudo)canónicos activan el componente polar, los atenuantes, que lo desactivan, carecen de sustancia semántica (Cuadro 11):

\begin{tabular}{|l|l|l|c|}
\cline { 2 - 4 } \multicolumn{1}{l|}{ Casi } & COMPONENTE POLAR & INVERSIÓN POLAR & SUSTANCIA SEMÁNTICA \\
\hline (pseudo)canónico & activado & casi-P $\Rightarrow \neg \mathrm{P}^{\prime}$ & SÍ \\
\hline Atenuante & desactivado & casi-P $\Rightarrow \mathrm{P}^{\prime}$ & $\emptyset$ \\
\hline
\end{tabular}

Cuadro 11. Polaridad y sustancia semántica.

Los aproximativos, como tal, carecerían de sustancia semántica (Horn 2011) pero, mientras que los usos (pseudo)canónicos la adquieren para su componente polar, los usos atenuantes permanecen desactivados. De manera que la activación o desactivación del componente polar depende de su naturaleza semántica. Esto, sin embargo, no puede constatarse ni por su posición sintáctica, que coincide en (14"a) y (14"b); ni por su contexto, que puede omitirse en ambos casos.

Para explicar este mecanismo de activación y desactivación polar, se propone aquí equiparar el concepto de sustancia semántica (no definida en la bibliografía deductiva) con el de contenido proposicional (Cuadro 12):

\begin{tabular}{|l|l|l|c|}
\cline { 2 - 4 } \multicolumn{1}{c|}{ Casi } & COMPONENTE POLAR & INVERSIÓN POLAR & CONTENIDO PROPOSICIONAL \\
\hline (pseudo)canónico & Activado & casi-P $\Rightarrow \neg \mathrm{P}^{\prime}$ & SÍ \\
\hline Atenuante & Desactivado & casi-P $\Rightarrow \mathrm{P}^{\prime}$ & $\emptyset$ \\
\hline
\end{tabular}

Cuadro 12. Polaridad y contenido proposicional. 
La idea de sustancia semántica suele emplearse para el lexema de una palabra, mientras que contenido proposicional se refiere al significado mínimo de una expresión compuesta (Dowty et alii 1981). Sin embargo, el hecho de que casi adquiera sustancia semántica implica que esta forma pueda constituir por si sola el contenido mínimo de una intervención en la conversación coloquial ${ }^{5}$.

La noción de contenido proposicional permite relacionar la naturaleza semántica de casi con el tipo de unidad discursiva que este aproximativo ocupa según el modelo Val.Es.Co. Las unidades con contenido proposicional son aquellas que "tienen sustancia semántica" (Grupo Val.Es.Co. 2014: 53), mientras que las unidades sin contenido proposicional dependen de una unidad jerárquicamente superior (Cuadro 13):

\begin{tabular}{|l|l|l|l|}
\cline { 2 - 4 } \multicolumn{1}{l|}{ Casi } & COMPONENTE POLAR & INVERSIÓN POLAR & \multicolumn{1}{c|}{ UNIDADES } \\
\hline (pseudo)canónico & Activado & casi-P $\Rightarrow \neg \mathrm{P}^{\prime}$ & $\begin{array}{l}\text { Subacto sustantivo director (SSD) } \\
\text { Subacto sustantivo subordinado (SSS) }\end{array}$ \\
\hline Atenuante & Desactivado & casi-P $\Rightarrow \mathrm{P}^{\prime}$ & Subacto adyacente (SA) \\
\hline
\end{tabular}

Cuadro 13. Polaridad y unidades.

La unidad informativa superior, el subacto sustantivo director (SSD), se ajusta al principio de aislabilidad e identificabilidad, pues puede funcionar de forma adecuada en el discurso independientemente de las otras unidades que la acompañan. Es decir, puede "funcionar sola constituyendo una intervención” (Pons 2016: 548). Un ejemplo ad hoc sería:

(16) $\#\{$ SSD Casi gané la lotería SSD $\} \#$

que podemos aislar como constituyente de una hipotética intervención independiente:

(16') B': ¿Ganaste la lotería?

A': \#\{SSD casi SSD $\} \#$

Por su parte, las unidades jerárquicamente inferiores, los subactos adyacentes (SA), no son aislables (17'), ya que su contenido proposicional depende del subacto sustantivo (ss) al cual se adscriben:

(17) \#\{SSD Casi paga tú SSD $\}\{$ SSS y luego hacemos cuentas SSS $\} \#$

(17') B': Si te parece, pago yo.

A': ??\#\{SSD casi SSD $\} \#$

En el supuesto de situar este uso de casi en una intervención distinta de la de (17), es necesario, en cualquier caso, otro elemento lingüístico que dote al SSD de contenido proposicional:

(17") B': Si te parece, pago yo.

A': \#\{SSD casi sí SSD $\} \#$

A": \#\{SSD sí SSD $\}$

5 En efecto, como bien señala un revisor anónimo, sustancia semántica y contenido proposicional son conceptos diferentes. Sin embargo, pueden relacionarse mediante el criterio de aislabilidad, en la medida en que un elemento con sustancia semántica puede aislarse en tanto que constituyente mínimo necesario para una proposición. Algo imposible con el casi atenuador. 
En conclusión, la hipótesis de partida queda como sigue: casi presenta un componente polar desactivado si la unidad que ocupa carece de contenido proposicional (Cuadro 14),

\begin{tabular}{|l|c|c|c|}
\cline { 2 - 4 } \multicolumn{1}{c|}{} & COMPONENTE POLAR & CONTENIDO PROPOSICIONAL & UNIDAD \\
\hline casi (pseudo)canónico & $\neg \mathrm{P}^{\prime}$ & SÍ & $\begin{array}{c}\text { SSD } \\
\text { SSS }\end{array}$ \\
\hline casi atenuante & & & SA \\
\hline
\end{tabular}

Cuadro 14. Hipótesis (esquema).

lo cual permite distinguir, desde la sintaxis discursiva, los usos atenuantes de los usos (pseudo)canónicos.

\subsection{Segunda hipótesis: casi como SAM}

Los usos atenuantes tienen un valor pragmático. La desactivación de su componente polar, así como la función pragmática derivada del componente próximo descendente, hacen del casi atenuante un elemento modalizador propio de los enunciados de la cortesía comunicativa (vid. § 2.1.2):

(18) Casi paga tú.
a. CPRox $(\downarrow \mathrm{P}):(\mathrm{M}+>)$ distanciamiento
b. $(++>)$ disminución de la fuerza ilocutiva (= atenuación)

(18') Paga tú (= enunciado neutro).

Así, casi puede elidirse sin alterar el significado del resto del acto de habla (18'). Si aplicamos el criterio de aislabilidad, los usos atenuantes de casi ocuparían unidades carentes de contenido proposicional y no podrían constituir por sí mismos una intervención, como muestran los ejemplos de (17') frente a los de (16'). En la segmentación discursiva, cuando los usos atenuantes no se ajustan al principio de aislabilidad, constituyen sA, dado que su omisión no afecta significativamente al conjunto del acto (17').

Ello perfila la siguiente hipótesis final: en su uso atenuante, la unidad jerárquicamente inferior que ocupa casi en la sintaxis discursiva es la de subacto adyacente modalizador (SAM):

(18) A: \#\{SAM Casi SAM $\}\{$ SSD paga tú. SSD $\} \#$

(18') B: ¿pago yo?

A': \#\{SAM casi $\mathrm{SAM}\}\{$ SSD sí $\mathrm{SSD}\} \#$

A": \# Ø\{SSD sí SSD $\} \#$

A"”: ??\# $\{$ SAM casi SAM $\} \varnothing$ \#

El SAM es una unidad que "introduce algún tipo de matización modal específica (atenuación, intensificación) sobre el subacto sustantivo al que se adhieren" (Grupo Val.Es.Co. 2014: 60). Su falta de contenido proposicional coincide con la desactivación del componente polar (no invierte los valores de verdad) y su componente próximo descendente modaliza el enunciado al uso, al tiempo que sirve al hablante de estrategia comunicativa. 


\section{RESULTADOS}

Este trabajo pretende arrojar luz sobre la naturaleza semántica de casi en sus usos atenuantes. Nuestra hipótesis, según la cual es posible recurrir al funcionamiento de casi en el discurso para testar la teorización en torno a su componente polar, se apoya en un estudio de corpus (§ 4.1) así como en el análisis de algunos ejemplos (§ 4.2).

\subsection{Estudio de corpus}

Para la recopilación del corpus, tanto el tipo de muestra como las bases de datos limitan el análisis a un estudio de carácter micro. Las razones son dos:

a. En primer lugar, para estudiar casi en conversaciones coloquiales, se recurre tanto al Corpus de Conversaciones Coloquiales (Briz y Grupo Val.Es.Co. 2002) como al digitalizado Valesco 2.0 (Cabedo y Pons 2013). La transcripción detallada de las conversaciones garantiza una muestra real de su uso, si bien el número de ocurrencias es reducido (64 en total).

b. En segundo lugar, teniendo en cuenta la relevancia del análisis en unidades discursivas, es preferible no ampliar la muestra a lo escrito, pues la segmentación se aplica óptimamente a lo oral.

Este estudio, pues, no recoge una muestra absolutamente representativa de los diferentes usos de casi, sino que dispone ciertos casos de la lengua hablada con los que poder ejemplificar la hipótesis de partida (pese a las restricciones cuantitativas). En definitiva, se trata de un análisis inductivo pero de orden cualitativo, mediante el cual perfilar una intuición lingüística.

El corpus recoge, en total, 64 ocurrencias de casi en sus contextos conversacionales y analiza sus componentes próximo y polar, así como su unidad y posición discursiva (Cuadro 15):

\begin{tabular}{|l|l|}
\multicolumn{1}{c|}{} & FICHA \\
\hline Componente próximo ascendente/descendente \\
\hline Componente polar activado/desactivado \\
\hline Unidad \\
\hline Posición \\
\hline
\end{tabular}

Cuadro 15. Ficha de estudio.

Ello permite clasificar las ocurrencias de casi según la categorización de §2.1.2. Así, los resultados son los siguientes (Cuadro 16):

\begin{tabular}{|l|l|}
\hline \multicolumn{1}{|c|}{ Usos } & \multicolumn{1}{c|}{$f_{o}$} \\
\hline Canónico & 40 \\
\hline Pseudocanónico & 17 \\
\hline Atenuante & 3 \\
\hline Invertido & 4 \\
\hline
\end{tabular}

Cuadro 16. Frecuencia. 
Tal y como se sigue de las categorías canónico y pseudocanónico (Pons y Schwenter 2011) para los usos polares de casi, estos dos tipos son los más frecuentes. Por su parte, los usos atenuantes, en proporción con el resto de la muestra, son apenas representativos.

El análisis especifica también la(s) unidad(es) en que se ubica cada tipo de casi (Cuadro 17):

\begin{tabular}{|c|c|c|}
\hline Usos & $f_{o}$ & UNIDAD \\
\hline \multirow{2}{*}{ Canónico } & \multirow{2}{*}{40} & 37 SSD \\
\hline & & $3 \mathrm{SSS}$ \\
\hline \multirow{2}{*}{ Pseudocanónico } & \multirow{2}{*}{17} & $12 \mathrm{SSD}$ \\
\hline & & 5 SSS \\
\hline \multirow{2}{*}{ Atenuante } & \multirow{2}{*}{3} & 2 SSD?? \\
\hline & & 1 SSS ?? \\
\hline \multirow{2}{*}{ Invertido } & \multirow{2}{*}{4} & 4 SSD \\
\hline & & 0 SSS \\
\hline
\end{tabular}

Cuadro 17. Unidades.

En su uso atenuante, casi formaría parte de unidades jerárquicamente superiores (sSD/ Sss). Ahora bien, estas unidades poseen contenido proposicional, lo cual contradice la hipótesis según la cual el componente polar de casi se desactiva al no disponer de la sustancia semántica de aquella unidad en que se ubica. En otras palabras, el componente polar entrañado por casi se desactiva cuando la unidad que ocupa carece de contenido proposicional. Dicha unidad es el SAM y, sin embargo, los datos muestran, en una primera cuantificación, lo contrario (SSD/sSS??).

La solución a este problema pasa por extender el análisis de la unidad en que casi presenta un uso atenuante a la posición que ocupa dentro de esta. Luego (Cuadro 18):

\begin{tabular}{|c|c|c|c|}
\hline CLASE & $f_{0}$ & UNIDAD & POSICIÓN \\
\hline \multirow{2}{*}{ Canónico } & \multirow{2}{*}{38} & 35 SSD & $\begin{array}{l}12 \text { inicial } \\
\mathbf{2 2} \text { media } \\
\mathbf{1} \text { final }\end{array}$ \\
\hline & & $3 \mathrm{SSS}$ & $\begin{array}{l}2 \text { inicial } \\
1 \text { media } \\
0 \text { final }\end{array}$ \\
\hline \multirow{2}{*}{ Pseudocanónico } & \multirow{2}{*}{17} & $12 \mathrm{SSD}$ & $\begin{array}{l}0 \text { inicial } \\
5 \text { media } \\
9 \text { final }\end{array}$ \\
\hline & & $5 \mathrm{SSS}$ & $\begin{array}{l}0 \text { inicial } \\
2 \text { media } \\
1 \text { final }\end{array}$ \\
\hline \multirow{2}{*}{ Atenuante } & \multirow{2}{*}{5} & 3 SSD?? & $\begin{array}{l}1 \text { inicial } \\
1 \text { media } \\
0 \text { final }\end{array}$ \\
\hline & & 1 SSS?? & $\begin{array}{l}1 \text { inicial } \\
0 \text { media } \\
0 \text { final }\end{array}$ \\
\hline \multirow{2}{*}{ Invertido } & \multirow{2}{*}{4} & 4 SSD & \multirow{2}{*}{$\begin{array}{l}3 \text { inicial } \\
1 \text { media } \\
0 \text { final }\end{array}$} \\
\hline & & 0 SSS & \\
\hline
\end{tabular}

Cuadro 18. Posiciones. 
Es posible, no obstante, cuestionar la posición del casi atenuante dentro de unidades (en principio) jerárquicamente superiores ( $\mathrm{SSD} / \mathrm{sss})$.

Así, la relación posición-unidad no se aplica de igual modo en los usos pseudocanónicos que en los usos atenuantes. En los primeros, casi no constituye una unidad propia, puesto que su omisión modificaría el contenido proposicional de todo su acto. Su posición es la de un elemento como parte de subacto, pues su presencia afecta al conjunto de la forma lógica (entraña negación) (Cuadro 19):

\begin{tabular}{|l|l|c|}
\hline \multicolumn{1}{|c|}{ Usos } & \multicolumn{1}{|c|}{$f_{0}$} & Relación UNIDAD-POSICIÓN \\
\hline (Pseudo)canónico & 57 & Parte de SSD/SSS \\
\hline Atenuante & 3 & SAM \\
\hline
\end{tabular}

Cuadro 19. Relación unidad-posición.

Por su parte, el casi atenuador funcionaría sintácticamente como un elemento extraoracional y, a diferencia de los usos (pseudo)canónicos, sería constitutivo de una unidad discursiva propia (SAM), en aydacencia con otro subacto sustantivo al que se adhiere. Para ejemplificarlo, analizamos detalladamente algunas ocurrencias.

\subsection{Análisis de ocurrencias}

Como muestran las tablas, los usos (pseudo)canónicos son los mayoritarios. En el siguiente ejemplo, el componente polar de casi, que se integra en un subacto con contenido proposicional, está activado:

(19) D: [hombre pero eso no] hace falta que lo [argumentes]

A: \#\{SSS [hablando de temaa SSS $\}$ \{SSD casi me pego una hostia con el coche hoy] SSD $\} \#$ B: $\quad[(())$ te argumento porqué tienes más dinero sin tener $]$ novia $\uparrow$ que $((\mathrm{si} \rightarrow))$

(Corpus Valesco 2.0, Conversación 45, Intervenciones 67-69)

Al ocupar una unidad con contenido proposicional, casi adquiere la sustancia semántica de que carece; como muestra el hecho de que pueda funcionar aisladamente como constituyente de una hipotética intervención:

(19') A': \#\{SSS [hablando de temaa SSS \} \{SSD me pegué una hostia con el coche hoy] SSD $\} \#$ B': ¿te pegaste una hostia?

$\mathrm{A}^{\prime}$ : \#\{SSD casi SSD $\} \#$

Este criterio para los usos (pseudo)canónicos es igualmente aplicable con posiciones finales:

(20) B: fuiste $\uparrow /$ muchas $\uparrow$ clases/ \#((o sea- $))$ me- hombre [me pelaba] $=\ldots$

A: $[(($ es que $))]$

$\mathrm{B}:=\ldots \#\{$ SSD yo las mismas que tú casi SSD $\} \#$

B: después de febrero yo no-no fui ni al (( )) 
(20') B': hombre me pelaba las mismas que tú

A': ¿las mismas que yo?

B': \#\{SSD casi SSD $\} \#$

y también con posiciones medias:

(21) N: \#\{SAT pues SAT $\}\{$ SSD en esta semana me lo he encontrao casi $[$ to(do)s los días SSD $\} \#(())$

A: [su hermana $\uparrow-\mathrm{su}$ ] hermana llamó ayer/ su hermana llamó ayer [que-=]

I: [también $\uparrow$ ]

(Corpus Valesco 2.0, Conversación 2, Intervenciones 205-207)

(21') N': pues en esta semana me lo he encontrao todos los días

A': ¿todos los días?

N': \#\{SSD casi SSD $\} \#$

ya que, independientemente de donde se sitúen, son en cualquier caso parte de subacto (hipotéticamente se pueden aislar, pero no constituyen una unidad propia).

Por su parte, un ejemplo como el uso atenuante de casi en posición inicial (16) presenta este aproximativo como un sintagma en función de modificador oracional, el cual a nivel discursivo tiene ámbito sobre la totalidad del enunciado (intervención 284) que encabeza (22’):

(22) E: el Cádiz está ya acabao tío $\uparrow$

A: \#el Cádiz está en segunda be yaa/ tío \# \#\{SSD (// casi[ peor que-] SSD $\} \#^{6}$

E: [prácticamente]

A: puees de segunda bee/ a la desapariciónn / tiene ((ahí)) nada eh $\uparrow$

(Corpus Valesco 2.0, Conversación 14, Intervenciones 283-286)

(22’) A: \#el Cádiz está en segunda be yaa/ tío\# \#(// casi [SSD?? [peor que-] SSD??\}\#

No obstante, casi no invierte la polaridad en (22): $\{S S D$ casi [ peor que-] $S S D\} \Rightarrow$ 'El Cádiz sí está peor que el otro equipo elidido’. Su componente polar está desactivado pero, en una primera segmentación, la unidad en que teóricamente se inserta (SSD??) poseería contenido proposicional. Frente a esto, un segundo análisis que tenga en cuenta la falta de sustancia semántica en los aproximativos hace incompatible los usos atenuantes con el contenido proposicional. Ello obliga a una segmentación alternativa, según la cual casi constituye una unidad propia jerárquicamente inferior:

(22”) A: \#el Cádiz está en segunda be yaa/ tío\# \#\{SAM (//casi SAM $\}\{$ SSD [ peor que-] SSD $\} \#$

6 En este SSD, el acto \#casi[ peor que-]\# está truncado. El hablante A omite el segundo término de la comparación mediante la cual iba a contraponer la mala situación del Cádiz con la de otro equipo de fútbol. Sin duda, se trata de un contenido elidido, pero no por ello deja de constituir acto. Ello se ve claramente si rellenamos esta omisión con un contenido adecuado cualquiera:

$$
\begin{aligned}
& \text { - A: \#el Cádiz está en segunda be yaa\#\# \#SAI (/ tío SAI }\}\{\text { SSD (// casi[ peor que- ] SSD }\} \# \\
& \text { - A': } \\
& \text { \{SSD (// casi }[\text { peor que } \text { el Xerez] SSD }\} \#
\end{aligned}
$$

De hecho, recientes revisiones (Pons 2016) proponen la notación $\{S S X\}$ para estas unidades constituyentes de actos truncados. 
Entender esta posición de casi como un SAM (es decir, como unidad dependiente y modalizadora) explica tanto la ausencia de inversión polar (pues repercute sobre la proposición) como el valor atenuante que ejerce sobre el SSD al que se adhiere. Esta posición inicial del casi atenuante supone una unidad distinta (SAM) pero no independiente. Como atenuador, casi no puede constituir una intervención propia, como corrobora que pueda eliminarse sin que el resto de constituyentes del acto pierda coherencia:

(22”) A: \#el Cádiz está en segunda be yaa/ tío\# \#\{SAM (//casi SAM $\}$ SSD [ peor que el Xerez] SSD $\} \#$

(22”') A: \#el Cádiz está en segunda be yaa/ tío\# \#(//Ø\{SSD [ peor que el Xerez] SSD $\} \#$

De acuerdo con esto, existe una correlación entre el uso atenuante y el hecho de que casi no sea aislable, a lo cual se ajustan las otras ocurrencias del corpus. Tanto cuando casi constituye un SAM en posición inicial de sss:

(23) B: [...] lo que habíamos hecho había sido eso $\uparrow[\ldots]$ pues un cuarto de hora $\uparrow$ veinte minutos más $\downarrow$ hacer la [parte de=]

A: $\quad[\mathrm{mm}]$

$\mathrm{B}:=$ gramática $/ / /$

A: \#pues no sé $\downarrow \#$ \#\{SAT entonces SAT $\}\{$ SSD me parece que SSD $\}\{$ SAM casi SAM $\}$ $\{\mathrm{SSS}$ lo vo- voy a decirles que vengan UN día diferente/ SSS $\}$ SSS a todos los que estén en ese caso// SSS \}\# \#y les paso por ejemplo ell-// no el test que hicimos enn- en enero $\uparrow$ uno quee te voy a poner $\rightarrow \#$

B: esa es otra [solución]

(Corpus Valesco 2.0, Conversación 31, Intervenciones 159-163)

(23') A: \#pues no sé $\downarrow \#$ \#\{SAT entonces SAT $\}$ SSD me parece que SSD $\}$ Ø $\{$ SSS lo vo- voy a decirles que vengan UN día diferente/ SSS $\}\{\mathrm{SSS}$ a todos los que estén en ese caso// SSS $\} \#$ \#y les paso por ejemplo ell-// no el test que hicimos enn- en enero $\uparrow$ uno quee te voy a poner $\rightarrow \#$

como cuando el uso atenuante de casi constituye un SAM en posición media de SSD:

(24) A: = sí que ha dicho $\uparrow[\ldots]$ quee sí que nos dan certificadoo de horas $\uparrow[y y=]$

$\mathrm{B}:$

[ide asistencia?]

$\mathrm{A}:=$ sí§

B: \#\{SAT §pues SAT $\}\{$ SSD yo estoy $\{$ SAM casi SAM $\}$ en contra de eso SSD $\}\{$ SAI ¿eh?/ SAI\}\#

(Corpus Valesco 2.0, Conversación 31, Intervenciones 290-293)

(24') B: \#\{SAT §pues SAT $\}\{$ SSD yo estoy $\emptyset$ en contra de eso SSD $\}\{$ SAI ¿eh?/ SAI $\} \#$

en cuyo caso casi, aunque está insertado (24), sigue siendo modal; pues puede elidirse (24'), pese a una notable reducción del efecto atenuante que ejerce sobre el conjunto del acto.

7 Rellenamos aquí, para una mejor ejemplificación, la elisión original de A en la intervención 284, ej. (22”). 


\section{CONCLUSIONES}

Este trabajo dispone una organización deductivo-inductiva. Se han expuesto los principales problemas teóricos a la hora de abordar los usos atenuantes de casi, así como las dos principales líneas de análisis. Esto, a su vez, se ha tratado de complementar mediante la segmentación de ejemplos extraídos de conversaciones coloquiales. Podemos esbozar las siguientes conclusiones:

a. Disponer de un sistema de unidades permite observar los usos atenuantes de casi como parte de su significado (no como excepciones contextuales).

b. La unidad SAM redefine el denominado casi expletivo como un atenuador.

c. La atenuación al uso mediante aproximativos no depende de interpretaciones cognitivas de los hablantes, sino que tiene un funcionamiento sistemático.

d. Casi es un elemento bicategorial: ha de analizarse en función del nivel lingüístico en que funciona (desde su integración proposicional o como una unidad discursiva suprasintáctica).

e. El estudio de la atenuación en casi implementa la investigación en aproximativos: concreta el concepto de sustancia semántica recurriendo a su equiparación con la noción de contenido proposicional en las unidades discursivas, al tiempo que valida la tesis del entrañamiento asertóricamente inerte.

Finalmente, los resultados del presente trabajo ponen de relieve la necesidad de una orientación discursiva para la investigación en aproximativos.

\section{Referencias bibliográficas}

Albelda, M. (2005). “Aportaciones del operador modal fijo al estudio de la intensificación”. En Bernabé, A., Berenguer, J.A., Cantarero, M. y J.C. de Torres. Actas del XXX Congreso de la Sociedad Española de Lingüística. Madrid: Gredos, pp. 596-513.

Albelda, M. (2010). “¿Cómo se reconoce la atenuación?”. En Orletti, F. y L. Mariottini (eds.). (Des) cortesía en español. Espacios teóricos y metodológicos para su estudio. Roma: Università Roma Tre, pp. 47-70.

Amaral, P. (2006). "On the semantics of Almost". LSA Annual Meeting (6-1-2006).

Amaral, P. (2007). The Meaning of Approximative Adverbs: Evidences from European Portuguese. Tesis Doctoral, Universidad Estatal de Ohio.

Amaral, P. y F. del Prete (2010). "Approximating the limit: the interaction between quasi 'almost' and some temporal connectives in Italian”, Linguist and Philos, 33, pp. 51-115.

Aranovich, R. (1995). "Spanish Casi as Scalar Operator". En Bilmes, L., Liang, A.C. y W. Ostapirat (eds.). Proceedings of the Twenty-First Annual Meeting of the Berkeley Linguistics Society, pp. 12-23.

Briz, A. y Grupo Val.Es.Co. (2002). Corpus de conversaciones coloquiales. Anejo de la revista Oralia. Madrid: Arco Libros.

Briz, A. (2007). "Para un análisis semántico, pragmático y sociopragmático de la cortesía atenaudora en España y América", Lingüística Española Actual, XXIX/1, pp. 5-44.

Briz, A. y S. Pons (2010). “Unidades, marcadores discursivos y posición”. En Loureda, Ó. y E. Acín (eds.). Los estudios sobre marcadores del discurso, hoy. Madrid: Arco/Libros, pp. 523-557.

Cabedo Nebot, A. y S. Pons Bordería (2013). Corpus Valesco 2.0.: www.valesco.es (20/11/2016). 
De Miguel, E. (1999). “El aspecto léxico”. En Bosque, I. y V. Demonte (eds.). Gramática descriptiva de la lengua española. Vol. II. Madrid: Espasa Calpe, pp. 2977-3060.

Dowty, D., Wall, R. E. y S. Peters (1981). Introduction to Montague Semantics. Dordrecht: Reidel Publishing Company.

Ducrot, O. (1982). Decir y no decir. Hachette: Buenos Aires.

Estellés, M. y S. Pons (2009). "Expressing digression linguistically. Do digressive markers exist?", Journal of Pragmatics, 4(51), pp. 931-936.

Estellés, M. (2011). Gramaticalización y paradigmas: un estudio a partir de los denominados marcadores de digresión en español. Frankfurt am Main: Peter Lang.

Fernández Leborans, Mª.J. (1999). "La predicación: las oraciones copulativas". En Bosque, I. y V. Demonte (eds.). Gramática descriptiva de la lengua española. Vol. II. Madrid: Espasa Calpe, pp. 2357-2460.

Grice, H.P. (1975). "Logic and Conversation". En Cole, P. y J.L. Morgan (eds.). Syntax and semantics 3: Speech acts. New York: Academic Press, pp. 41-58.

Grupo Val.Es.Co. (2014). "Las unidades del discurso oral. La propuesta Val.Es.Co. de segmentación de la conversación (coloquial)", Estudios de Lingüística del Español, 35, pp. 13-73.

Horn, L. (2002). "Assertoric inertia and NPI licensing”, CLS, 38(2), pp. 55-82.

Horn, L. (2009). “Almost et al.: scalar adverbs revisited”. En Lee, C., Kiefer, F. y M. Krifka (eds.). Current issues in unity and diversity of languages. Cham: Springer, pp. 283-304.

Horn, L. (2011). "Almost forever”. En Yuasa, E., Bagchi, T. y K. Beals (eds.). Pragmatics and autolexical grammar. In honor of Jerry Saddock. Amsterdam/Philadelphia: John Benjamins, pp. 1-21.

Kovacci, O. (1999). "El adverbio". En Bosque, I. y V. Demonte (eds.). Gramática descriptiva de la lengua española. Vol. I. Madrid: Espasa Calpe, pp. 705-786.

Levinson, S. (2000). Presumptive Meanings. The Theory of Generalized Conversational Implicature. Cambridge MA: MIT Press.

Li, C. (1976). "A functional Explanation for an Unexpected Case of Ambiguity (S or $\sim S)$ ". En Juilland, A. (ed.). Linguistics Studies offered to Joseph Greenberg on the Occasion of his Sixtieth Birthday. Saratoga: Anma Libri, pp. 527- 535

Narbona Jiménez, A. (1988). "Sintaxis coloquial: problemas y métodos", Lingüística Española Actual, 1, pp. 81-106.

Pons, S. (2016). “Cómo dividir una conversación en actos y subactos”. En Bañón Hernández, A.M., Espejo Muriel, M. ${ }^{a}$ M., Herrero Muñoz-Cobo, B. y J. L. López Cruces (eds.). Oralidad y análisis del discurso. Homenaje a Luis Cortés Rodríguez. Almería: Editorial Universidad de Almería, pp. 545-566.

Pons, S. y S. Schwenter (2011). "Los significados próximo y polar de casi". En González Ruiz, R. y C. Llamas Saíz (eds.). Gramática y discurso. Nuevas aportaciones sobre partículas discursivas del español. Pamplona: EUNSA, pp. 159-188.

Sadock, J. (1981). “Almost”. En Cole, P. (ed.). Radical Pragmatics. New York: Academic Press, pp. 257-272.

Salameh Jiménez, S. (2014). Aproximaciones al estudio de subjetividad e intersubjetividad en marcadores discursivos: relaciones entre periferias, marcadores discursivos y posiciones. Trabajo final de grado, Universitat de València.

Schwenter, S. (2002). "Discourse context and polysemy: Spanish casi", Romance Philology and Variation: Selected Papers from the 30th Linguistic Symposium on Romance Languages, págs. 161-175.

Sevi, A. (1998). A semantics for 'Barely' and 'Almost'. Disertación de máster, Universidad de Tel-Aviv.

Ziegeler, D. (2000). "What can almost reveal about counterfactual inferences", Journal of Pragmatics, 32, pp. 1743-1776.

Ziegeler, D. (2010). "Running the gauntlet on the approximatives debate: A response to recent challenges", Journal of Pragmatics, 42, pp. 681-704.

Ziegeler, D. (2016). "Intersubjectivity and the diachronic development of counterfactual almost", Journal of Historical Pragmatics, 17(1), pp. 1-25. 